\title{
What artificial grammar learning reveals about the neurobiology of syntax
}

\author{
Karl-Magnus Petersson ${ }^{\text {a,b,c,d,* }}$, Vasiliki Folia ${ }^{\text {a,b,c }}$, Peter Hagoort ${ }^{\text {a,b }}$ \\ a Max Planck Institute for Psycholinguistics, P.O. Box 310, NL-6500 AH Nijmegen, The Netherlands \\ ${ }^{\mathrm{b}}$ Donders Institute for Brain, Cognition and Behaviour: Center for Cognitive Neuroimaging, Radboud University Nijmegen, The Netherlands \\ ${ }^{\mathrm{c}}$ Cognitive Neurophysiology Research Group, Stockholm Brain Institute, Karolinska Institutet, Stockholm, Sweden \\ ${ }^{\mathrm{d}}$ Cognitive Neuroscience Research Group, Institute for Biotechnology E Bioengineering/CMBE, Universidade do Algarve, Faro, Portugal
}

\section{A R T I C L E I N F O}

Article history:

Available online 12 October 2010

\section{Keywords:}

FMRI

Syntax

Natural language

Artificial language

Broca's area

Artificial grammar learning

Adaptive dynamical systems

Computability

Chomsky hierarchy

Complexity

\begin{abstract}
A B S T R A C T
In this paper we examine the neurobiological correlates of syntax, the processing of structured sequences, by comparing FMRI results on artificial and natural language syntax. We discuss these and similar findings in the context of formal language and computability theory. We used a simple right-linear unification grammar in an implicit artificial grammar learning paradigm in 32 healthy Dutch university students (natural language FMRI data were already acquired for these participants). We predicted that artificial syntax processing would engage the left inferior frontal region (BA 44/45) and that this activation would overlap with syntax-related variability observed in the natural language experiment. The main findings of this study show that the left inferior frontal region centered on BA 44/45 is active during artificial syntax processing of well-formed (grammatical) sequence independent of local subsequence familiarity. The same region is engaged to a greater extent when a syntactic violation is present and structural unification becomes difficult or impossible. The effects related to artificial syntax in the left inferior frontal region (BA 44/45) were essentially identical when we masked these with activity related to natural syntax in the same subjects. Finally, the medial temporal lobe was deactivated during this operation, consistent with the view that implicit processing does not rely on declarative memory mechanisms that engage the medial temporal lobe. In the context of recent FMRI findings, we raise the question whether Broca's region (or subregions) is specifically related to syntactic movement operations or the processing of hierarchically nested non-adjacent dependencies in the discussion section. We conclude that this is not the case. Instead, we argue that the left inferior frontal region is a generic on-line sequence processor that unifies information from various sources in an incremental and recursive manner, independent of whether there are any processing requirements related to syntactic movement or hierarchically nested structures. In addition, we argue that the Chomsky hierarchy is not directly relevant for neurobiological systems.
\end{abstract}

(c) 2010 Elsevier Inc. All rights reserved.

\section{Introduction}

The human capacity for language and communication is subserved by a network of brain regions that collectively instantiate the semantic, syntactic, phonological and pragmatic operations necessary for adequate language comprehension and production. A growing number of studies on the neural architecture of language, using electromagnetic (EEG/MEG) and hemodynamic methods (PET/FMRI), have added to, and also changed previous views on the brain's infrastructure for language. Before elaborating on some current issues related to the neurobiology of syntax, here is what we believe to be the major conclusions from the overall body of literature on the neurobiology of language:

\footnotetext{
* Corresponding author. Tel.: +31 2436 10984; fax: +31 243610652.

E-mail address: karl-magnus.petersson@mpi.nl (K.-M. Petersson).
}

(i) The language network is more extended than the classical language regions (Broca's and Wernicke's areas). It includes, next to Broca's region, adjacent cortex in the left inferior and middle frontal region, as well as substantial parts of superior and middle temporal cortex, inferior parietal cortex, and parts of the basal ganglia. In addition, homotopic regions in the right hemisphere are more often than not engaged in language processing (Hagoort, 2009).

(ii) The division of labor between Broca's region (frontal cortex) and Wernicke's region (temporal cortex) is not language production vs. language comprehension. The neocortex centered on the left inferior frontal region is involved in, at least, syntactic and semantic unification (on-line combinatorial operations during comprehension). Wernicke's region is involved in language production, at least at the level of word-form encoding (Indefrey \& Levelt, 2004). 
(iii) None of the language-relevant regions and none of the language-relevant neurophysiological effects are language-specific. All language-relevant ERP effects (e.g., N400, P600, (E)LAN) seem to be triggered by other than language input as well (e.g., music, pictures, gestures).

(iv) For language, as for most other cognitive functions, the function-to-structure mapping as one-area-one-function is almost certainly incorrect. More likely, any cortical region is a node that participates in the function of more than one functional network. Conceivably, top-down connections from supramodal regions could differentially recruit such a cortical node in the service of one network or another (Mesulam, 1990, 1998).

In normal language processing, semantics, phonology and syntax operate in close spatial and temporal contiguity in the human brain. Therefore the artificial grammar learning (AGL) paradigm has been used to create a relatively uncontaminated window onto the neurobiology of syntax (Gómez \& Gerken, 2000; Petersson, Forkstam, \& Ingvar, 2004; Reber, 1967). In addition, AGL has been used in cross-species comparisons in an attempt to establish the uniquely human component of language (Fitch \& Hauser, 2004; Gentner, Fenn, Margoliash, \& Nusbaum, 2006; Hauser, Chomsky, \& Fitch, 2002; O’Donnell, Hauser, \& Fitch, 2005; Saffran et al., 2008). Here, we will present data from an FMRI experiment that speaks to the neurobiology of syntax. In addition, we will discuss some of the theoretical issues resulting from the fact that, from a brain perspective, reference to and application of the Chomsky hierarchy is not directly relevant - a point that can be made on linguistic grounds as well (Pullum \& Scholz, 2009, 2010).

The implicit AGL paradigm allows a systematic investigation of aspects of structural (i.e., syntactic) acquisition from grammatical examples alone, without providing explicit feedback, teaching instruction, or engaging the subjects in explicit problem solving based on instruction. These acquisition conditions resemble, in certain important respects, those found in natural-language development. Generally, AGL consists of acquisition and test phases. In the acquisition phase, participants are exposed to an acquisition sample generated from a formal grammar. In the standard version, subjects are informed after acquisition that the sequences were generated according to a complex set of rules, and are asked to classify novel sequences as grammatical or not, based on their immediate intuitive impression (i.e., guessing based on "gut feeling"). A robust finding in this type of paradigm is that after several days of implicit acquisition subjects perform well above chance; they do so on regular (e.g., Folia et al., 2008; Forkstam, Elwér, Ingvar, \& Petersson, 2008; Petersson et al., 2004; Stadler \& Frensch, 1998 ) as well as non-regular grammars (Poletiek, 2002; Uddén et al., 2009), generating context-free and context-sensitive non-adjacent dependencies (Uddén, Ingvar, Hagoort, \& Petersson, submitted for publication; Uddén et al., 2009). In passing, we note that a qualitative match between the performance of simple recurrent networks and human comprehension of nested (context-free) and crossed (context-sensitive) dependencies has been reported (Christiansen \& Chater, 1999; Christiansen \& MacDonald, 2009). Because (in a technical sense), noisy or discrete simple recurrent networks are finite-state architectures (Casey, 1996; Maass, Joshi, \& Sontag, 2007; Maass \& Orponen, 1998; Maass \& Sontag, 1999; see also, Petersson, 2005b; Petersson, Grenholm, \& Forkstam, 2005), these results suggest that actual language processing uses no more on-line memory resources than can be provided by a finite-state architecture. These simulations, of course, only illustrate that recurrent networks can handle (bounded) non-regular processing at some level of proficiency. However, a correlation between the processing of long-distance-dependencies in natural language and statistical learning of non-adjacent dependencies was recently reported, suggesting a link between natural-language processing and implicit sequence learning. The latter performance was adequately modeled by a simple recurrent network in a visuomotor sequence learning task (Misyak, Christiansen, \& Tomblin, 2009, 2010).

The recursion-only hypothesis concerning the faculty of language (Hauser et al., 2002), and subsequent discussion (e.g., Chomsky, Fitch, \& Hauser, 2005; Jackendoff \& Pinker, 2005; Pinker \& Jackendoff, 2005), has inspired research on the neurobiology of syntax to be phrased in terms of recursion and the Chomsky hierarchy. More specifically, the recursion-only hypothesis suggests that some aspects of the language faculty are shared with non-human animals, whereas other aspects are specific to the human language faculty and the quest for "core syntax" in behavioral and functional neuroimaging studies of natural and artificial syntax has sometimes centered on the theoretical construct of the Chomsky hierarchy (Fig. 1).

In particular the syntactic feature of center- or nested embedding has been the focus of recent research (Bahlmann, Schubotz, \& Friederici, 2008; Fitch \& Hauser, 2004; Friederici, Bahlmann, Heim, Schubotz, \& Anwander, 2006; Makuuchi, Bahlmann, Anwander, \& Friederici, 2009). In the linguistic and psycholinguistic literature, the Chomsky hierarchy is most often formulated in terms of formal grammars. However, from a neurobiological point of view it is more natural to formulate the Chomsky hierarchy in terms of (equivalent) computational mechanisms (cf. e.g., Cutland, 1980; Davis, Sigal, \& Weyuker, 1994; Hopcroft, Motwani, \& Ullman, 2000; Savage, 1998; Soare, 1996), since the objective in neurobiology is to identify the neurobiological mechanisms underlying syntax. From the point of view of computability theory, the Chomsky hierarchy is in essence a memory hierarchy, which specifies the necessary (minimal) memory resources required for a given level of computational expressivity. However, it is not a complexity hierarchy for the mechanism(s) involved in various computational architectures, which are all equivalent to finite-state architectures (Minsky, 1967; Soare, 1996; Turing, 1936a, 1936b; Wells, 2005). We will return to the significance of this fact in the discussion section.

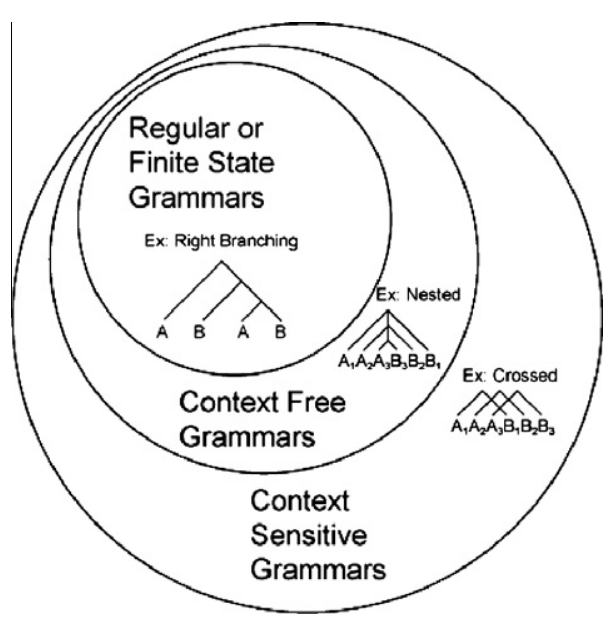

Fig. 1. The Chomsky hierachy. Informally, regular (finite-state) or right-linear phrase-structure grammars are built from a collection of production rules of the form $S \rightarrow$ abS and $S \rightarrow$ ab (where lower case indicates terminal symbols and $S$ a non-terminal sentence or start symbol). It is the inclusion of the start symbol on the right hand side of the first regular rule $(S \rightarrow$ abS) that makes this grammar recursive (Soare, 1996). The non-regular context-free case allows the right hand side to involve terminal symbols around the sentence symbol additional as in $\mathrm{S} \rightarrow \mathrm{aSb}$ and $\mathrm{S} \rightarrow \mathrm{ab}$. In the non-regular context-sensitive case, the left hand side has a "context" as exemplified in $a_{1} a^{n} S_{b_{1}} b^{n} \rightarrow a_{1} a^{n} a_{n+2} S b_{1} b^{n} b_{n+2}$ (cf., Davis et al., 1994). 
In this event-related FMRI study, we investigated a simple rightlinear unification grammar (cf., Hagoort, 2005; Joshi \& Schabes, 1997; Vosse \& Kempen, 2000). In an implicit AGL paradigm 32 subjects were exposed to grammatical sequences during an immediate short-term memory task. No performance feedback was provided. Implicit acquisition took place over 5 days. On the last day a grammaticality classification test was administered, in which new sequences were presented in a $2 \times 2$ factorial design, with the factors grammaticality and local subsequence familiarity (Forkstam, Hagoort, Fernandez, Ingvar, \& Petersson, 2006; Knowlton \& Squire, 1996; Meulemans \& Van der Linden, 1997). The second factor, local subsequence familiarity (technically, associative chunk strength (ACS), cf., Forkstam et al., 2006; Meulemans \& Van der Linden, 1997), is an associative measure of the superficial resemblance between classification sequences and the sequences in the acquisition set. The classification sequences with high local subsequence familiarity contained subsequences that appeared frequently in the acquisition set, while sequences with low local subsequence familiarity contained subsequences of low frequency in the acquisition set. In addition, natural language data was acquired in the same subjects in another FMRI experiment, very similar to the artificial grammar experiment, in a $2 \times 2$ factorial design with the factors syntax and semantics (Folia, Forkstam, Hagoort, \& Petersson, 2009). Based on previous findings (Forkstam et al., 2006; Petersson et al., 2004; Uddén et al., 2008), we predicted that artificial syntax processing would engage the left inferior frontal region (Brodmann's area (BA) 44 and 45) and that this activation would overlap with syntax-related variability observed in the natural language experiment.

In the context of recent FMRI findings, we raise the question whether Broca's region (or subregions) is specifically related to syntactic movement operations or the processing of hierarchically nested non-adjacent dependencies in the discussion section. We conclude that this is not the case. Instead, we argue that the left inferior frontal region is a generic on-line sequence processor that unifies information from various sources in an incremental and recursive manner, independent of whether there are any processing requirements related to syntactic movement or hierarchically nested structures.

\section{Materials and methods}

\subsection{Participants}

Thirty-two healthy right-handed (16 females, mean age $\pm S D=22 \pm 3$ years; mean years of education $\pm S D=16 \pm 2$ ) Dutch university students participated in the study. They were all pre-screened and none of the subjects used any medication, had a history of drug abuse, head trauma, neurological or psychiatric illness, or a family history of neurological or psychiatric illness. All subjects had normal or corrected-to-normal vision. Written informed consent was obtained from all participants according to the Declaration of Helsinki. Approval of the local medical ethics committee was obtained.

\subsection{The natural language experiment}

Here we briefly summarize the natural language experiment (for details, see Folia et al., 2009). The stimulus material consisted of 160 sentences from Hagoort (2003). The material consisted of sentence frames with a critical word position. There were four versions of sentence, one for each factor level combination (Table 1): (1) syntactically and semantically well-formed, correct sentences (CR); (2) semantically correct sentences with a gender agreement violation between the definite article and the noun (SY); (3) syn-
Table 1

Example sentences used in the natural language experiment. The critical noun is italicized, incorrect articles and anomalous adjectives are in bold. $\mathrm{CR}=$ correct; $\mathrm{SY}=$ syntactically anomalous; $\mathrm{SE}=$ semantically anomalous; and $\mathrm{CB}=$ combined syntactic and semantic anomalies.

$\begin{array}{ll}\text { De kapotte paraplu staat in de garage } & \text { (CR) } \\ \text { Het kapotte paraplu staat in de garage } & \text { (SY) } \\ \text { De eerlijke paraplu staat in de garage } & \text { (SE) } \\ \text { Het eerlijke paraplu staat in de garage } & \text { (CB) } \\ \text { The }_{\text {com }} / \text { The }_{\text {neut }} \text { broken/honest } \text { umbrella }_{\text {com }} \text { is in the garage } & \end{array}$

tactically correct sentences including a lexical semantic anomaly that consisted of a semantically unacceptable combination of the adjective and the following noun (SE); and (4) a combination of the syntactic and semantic anomalies (CB) described in (2) and (3). In the semantically correct and anomalous conditions, different adjectives preceded the nouns in the critical word (CW) position. These adjectives were matched in length and frequency. Critically, the violation of the gender agreement and the violation of the semantic constraint became clear at the same noun in critical word position. Thus, lexical differences do not interfere with the experimentally manipulated factors.

The sentence materials were constructed so that $25 \%$ of the sentences were syntactically and semantically correct; $25 \%$ contained a syntactic but no semantic anomaly; $25 \%$ contained a semantic but no syntactic anomaly; and $25 \%$ of the sentences contained both a syntactic and a semantic anomaly. To make sure that the violations of gender agreement did occur with equal probability after a common and neuter gender article, 160 filler sentences were added. In this way, violations of gender agreement could not be predicted on the basis of probability or sentence context. The sentences had a mean length of eight words ( $S D=1$ word). Words were never longer than 12 letters, with nouns in the $\mathrm{CW}$ position having a maximal length of 10 letters. All sentences were simple active or passive sentences. In the semantically correct/anomalous conditions, different adjectives preceded the nouns in the critical word position. These adjectives were matched in length and frequency. The experimental procedures were essentially identical to the AGL experiment (cf., below). The participants were instructed to read the sentences carefully and attentively for comprehension and to indicate for each sentence whether it was acceptable or not. Before the FMRI experiment started, each subject practiced on practice sentences to familiarize with the experimental procedure. The experimental sentences were presented in four blocks of approximately $10 \mathrm{~min}$ each, with a short break between each block.

\subsection{Stimulus material}

We used a simple right-linear unification grammar $\mathrm{G}$ with the following vocabulary of terminal symbols $\{\mathrm{M}, \mathrm{S}, \mathrm{V}, \mathrm{R}, \mathrm{X}\}$ and lexicon of primitive trees (treelets) $\left\{\left[\mathrm{s}_{1},\left[\mathrm{M}, \mathrm{s}_{2}\right]\right],\left[\mathrm{s}_{2},\left[\mathrm{~S}, \mathrm{~s}_{2}\right]\right],\left[\mathrm{s}_{2},\left[\mathrm{~V}, \mathrm{~s}_{4}\right]\right],\left[\mathrm{s}_{3}\right.\right.$, $\left.\left[X, s_{2}\right]\right],\left[s_{3},\left[X, s_{5}\right]\right],\left[s_{4},\left[R, s_{3}\right]\right],\left[s_{4},\left[S, s_{6}\right]\right],\left[s_{4}, \#\right],\left[s_{5},\left[R, s_{5}\right]\right],\left[s_{5},[M\right.$, $\left.\left.\left.s_{6}\right]\right],\left[s_{5}, \#\right],\left[s_{6}, \#\right]\right\}$. For a given lexical item (e.g., $\left.\left[s_{j},\left[T, s_{k}\right]\right]\right), s_{j}, s_{k}$ can be interpreted as syntactic control features and $\mathrm{T}$ as a surface feature. Within the unification framework (cf., Hagoort, 2005; Vosse \& Kempen, 2000), an incoming sequence of surface symbols (e.g., MSV ...) initiates the retrieval of lexical items from the mental lexicon. As a result, they enter an on-line unification space, $\left[\mathrm{s}_{1},[\mathrm{M}\right.$, $\left.\left.\mathrm{s}_{2}\right]\right],\left[\mathrm{s}_{2},\left[\mathrm{~S}, \mathrm{~s}_{2}\right]\right],\left[\mathrm{s}_{2},\left[\mathrm{~V}, \mathrm{~s}_{4}\right]\right] \ldots$, where two lexical items (e.g., $\left[\mathrm{s}_{i},[\mathrm{R}\right.$, $\left.\left.\left.s_{j}\right]\right],\left[s_{k},\left[Q, s_{l}\right]\right]\right)$ unify, combine, or merge through a unification operation $\mathrm{U}$ if and only if $\mathrm{s}_{j}=\mathrm{s}_{k}$, or $\mathrm{s}_{l}=\mathrm{s}_{i}$. This process is incremental and recursive: if the structure $\mathrm{U}\left(\left[\mathrm{s}_{1},\left[\mathrm{M}, \mathrm{s}_{2}\right]\right],\left[\mathrm{s}_{2},\left[\mathrm{~S}, \mathrm{~s}_{2}\right]\right]\right)=\left[\mathrm{s}_{1},\left[\mathrm{M},\left[\mathrm{s}_{2},[\mathrm{~S}\right.\right.\right.$, $\left.\left.\left.s_{2}\right]\right]\right]$ is already present in the unification space when the lexical item $\left[s_{2},\left[V, s_{4}\right]\right]$ is retrieved, a larger combinatorial structure can 
be formed by the unification operation $\mathrm{U}\left(\left[\mathrm{s}_{1},\left[\mathrm{M},\left[\mathrm{s}_{2},\left[\mathrm{~S}, \mathrm{~s}_{2}\right]\right]\right]\right],\left[\mathrm{s}_{2}\right.\right.$, $\left.\left.\left[\mathrm{V}, \mathrm{s}_{4}\right]\right]\right)=\left[\mathrm{s}_{1},\left[\mathrm{M},\left[\mathrm{s}_{2},\left[\mathrm{~S},\left[\mathrm{~s}_{2},\left[\mathrm{~V}, \mathrm{~s}_{4}\right]\right]\right]\right]\right]\right]$, and so on.

For this simple right-linear unification grammar, it is easy to construct the corresponding machine table (Minsky, 1967), here represented as a transition graph (Fig. 2). This is done by mapping the control features of a given lexical item $\left[\mathrm{s}_{j},\left[\mathrm{~T}, \mathrm{~s}_{k}\right]\right]$ onto corresponding internal states $s_{j}$, and $s_{k}$ of the computational machine, as well as onto a labeled transition $\mathrm{s}_{j} \stackrel{\mathrm{T}}{\rightarrow} \mathrm{s}_{k}$. We note that the Unification operator works similarly in all unification grammars. The structures generated by the Unification operator depend on the structure of the lexical items in any given formal language/grammar. Thus, in the current study, the Unification operator yielded right-linear phrase structures.

We generated 569 grammatical $(G)$ sequences from the grammar, with a sequence length ranging from 5 to 12 . For each item we calculated the frequency distribution of 2 and 3 letter chunks for both terminal and complete sequence positions. In this way we derived the associative chunk strength (ACS) for each item (cf., Forkstam et al., 2006; Knowlton \& Squire, 1996; Meulemans \& Van der Linden, 1997). Next, for the acquisition set we randomly selected in an iterative way 100 sequences that were representative, in terms the letter chunks, for the complete sequence set. In the next step, we generated the non-grammatical (NG) sequences, derived from non-selected $G$ sequences, by switching letters in two non-terminal positions. The NG sequences matched the $G$ sequences in terms of both terminal and complete-sequence ACS. Finally, in an iterative procedure, we randomly selected two sets of 56 sequences each from the remaining $G$ sequences, to serve as classification sets. These sets thus consisted of $25 \% \mathrm{G} /$ high ACS; $25 \%$ G/low ACS; $25 \%$ NG/high ACS; and 25\% NG/low ACS sequences.

In summary, the stimulus material included an acquisition set and two classification sets (all sets were pair-wise disjoint). The classification sets were used in a $2 \times 2$ factorial design with the factors Grammaticality and local subsequence familiarity (ACS). Thus, each classification set consisted of 28 sequences of each sequence-type: high ACS grammatical (HG), low ACS grammatical (LG), high ACS non-grammatical (HNG), and low ACS non-grammatical (LNG).

\subsection{Experimental procedures}

During the acquisition and classification sessions of the AGL part, each sequence was centrally presented letter-by-letter on a computer screen (3-7 s corresponding to 5-12 terminal symbols;

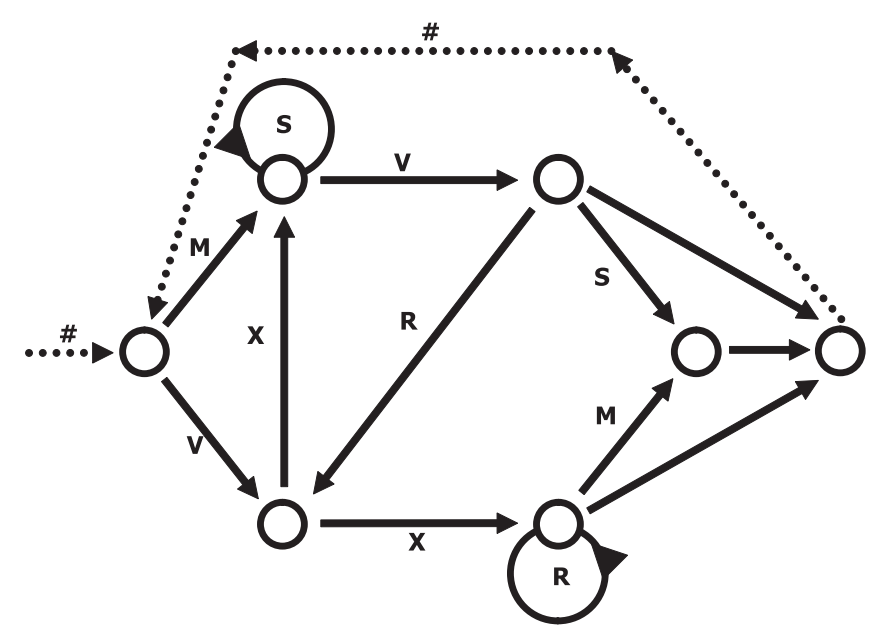

Fig. 2. The finite-state architecture parsing and producing the simple right-linear unification grammar used in the experiment, here represented as the corresponding transition graph implementation of the corresponding grammar G (Reber, 1967).
300 ms presentation, 300 ms inter-symbol-interval) using Presentation software (http://nbs.neuro-bs.com). Subjects were presented with the 100 acquisition sequences (presentation order randomized for each acquisition session), and the task was an immediate short-term memory task. When the last letter in a sequence disappeared, subjects were instructed to immediately reconstruct the sequence from memory and type it on a keyboard. No performance feedback was given, and only grammatical sequences (i.e., positive examples) were presented. The acquisition phase lasted approximately $20-40 \mathrm{~min}$. On the last (5th) day of the AGL experiment, subjects were informed about the existence of an underlying complex set of rules that determined the sequential structure of the sequences. Their new task was to classify novel sequences as grammatical or not, based on their immediate intuitive impression (i.e., guessing based on 'gut feeling'). This task was performed in the MRI-scanner. During classification, sequences were presented via an LCD-projector, projecting the computer display onto a semi-transparent screen that the subject comfortably viewed through a mirror device mounted on the head-coil, while FMRI data was acquired. The classification session was split in two parts, in order to balance response finger within subjects. Each part lasted approximately $20 \mathrm{~min}$. After a $1 \mathrm{~s}$ pre-stimulus period, the sequences were presented sequentially, followed by a $3 \mathrm{~s}$ response window. Within this response window, the subjects had to indicate their decision by pushing the corresponding response key with their left or right index finger. A low-level baseline condition was added, consisting of a sensorimotor decision task. In this task, sequences of repeated letters $P$ or $L$ (matched for sequence length to the classification items) were presented in the same fashion as the classification sequences. Subjects had to respond to these sequences by pressing the right or left index finger, respectively. The different stimulus types were presented in random order.

\section{Data acquisition and analysis}

Behavioral data were analyzed with repeated-measures ANOVAs (SPSS 15.0) with non-sphericity correction. A significance level of $P<0.05$ was used throughout.

\subsection{MR data acquisition}

Whole head $\mathrm{T}^{*}$-weighted functional echo planar blood oxygenation level dependent (EPI-BOLD) FMRI data were acquired with a SIEMENS Avanto 1.5T scanner using an ascending slice acquisition sequence (volume $\mathrm{TR}=2.6 \mathrm{~s}, \mathrm{TE}=40 \mathrm{~ms}, 90^{\circ}$ flip-angle, 33 axial slices, slice-matrix size $=64 \times 64$, slice thickness $=3 \mathrm{~mm}$, slice gap $=0.5 \mathrm{~mm}, \quad \mathrm{FOV}=224 \mathrm{~mm}$, isotropic voxel size $=3.5 \times 3.5 \times 3.5 \mathrm{~mm}^{3}$ ) in a randomized event related fashion. For the structural MR image volume, a high-resolution T1-weighted magnetization-prepared rapid gradient-echo pulse sequence was used (MP-RAGE; volume TR $=2250 \mathrm{~ms}$, TE $=3.93 \mathrm{~ms}$, $15^{\circ}$ flip-angle, 176 axial slices, slice-matrix size $=256 \times 256$, slice thickness $=1 \mathrm{~mm}$, field of view $=256 \mathrm{~mm}$, isotropic voxelsize $=1.0 \times 1.0 \times 1.0 \mathrm{~mm}^{3}$ ).

\subsection{MR image preprocessing and statistical analysis}

We used the SPM5 software for image preprocessing and statistical analysis. The EPI-BOLD volumes were realigned to correct for individual subject movement and were corrected for differences in slice-time. The subject-mean EPI-BOLD images were subsequently spatially normalized to the functional EPI template provided by SPM5. The normalization transformations were generated from the subject-mean EPI-BOLD volumes and applied to the corre- 
sponding functional volumes. The functional EPI-BOLD volumes were transformed into the MNI space, an approximate Talairach space (Talairach and Tournoux 1988), defined by the SPM5 template, and spatially filtered with an isotropic 3D spatial Gaussian filter kernel (FWHM $=10 \mathrm{~mm}$ ). The FMRI data were analyzed statistically, using the general linear model framework and statistical parametric mapping (Friston, Ashburner, Kiebel, Nichols, \& Penny, 2007) in a two-step mixed-effects summary-statistics procedure (Friston et al., 2007). We included the realignment parameters for movement artifact correction and a temporal high-pass filter (cycle cut-off at $128 \mathrm{~s}$ ), to account for various low-frequency effects.

At the first-level, single-subject fixed effect analyses were conducted. The linear model included explanatory regressors modeling the sequence presentation period from the violation position in the HNG and LNG conditions and their correct counterparts in the HG and LG conditions. This was done separately for correct and incorrect responses. The initial part of the sequences was also modeled separately, as was the baseline and the inter-sequenceinterval. The explanatory variables were temporally convolved with the canonical hemodynamic response function provided by SPM5. At the second-level, we generated single-subject contrast images for the correctly classified HG, LG, HNG, and LNG sequences, relative to baseline. These were analyzed in a random-effects repeated-measures ANOVA with non-sphericity correction for unequal variance between conditions. Statistical inference was based on the cluster-size test-statistic from the relevant second-level SPM[F] and SPM[T] maps thresholded at $P=0.001$ (uncorrected). Only clusters significant at $P<0.05$ (family-wise error (FWE) corrected for multiple non-independent comparisons based on smooth 3D random field theory, Adler, 1981; Adler \& Taylor, 2007; Friston et al., 2007; Worsley et al., 1996) are described. In the following, we use the terms activation and deactivation as synonyms for a relative increase and decrease in BOLD signal, respectively. For reasons of portability of results, we use the Talairach nomenclature (Talairach and Tournoux 1988) in the tables. In an additional analysis, we examined the common overlap between artificial and natural-syntax processing in the lateral prefrontal cortex by masking the NG vs. G effect with the natural-syntax-related variability that was observed in Folia et al. (2009), inclusive masking, mask thresholded at $P=.001$ ). Again, only clusters significant at $P<0.05$ family-wise error (FWE) corrected for multiple non-independent comparisons, based on smooth 3D random field theory, for the whole brain are reported. In addition, we list the coordinates of local maxima and their corresponding $P$-values corrected for the false discovery rate (FDR, Genovese, Lazar, \& Nichols, 2002) for descriptive purposes.

Finally, in a regional specific analysis, we tested for the nearest supra-threshold voxel (Friston, 1997; Worsley, 2003), and investigated spherical regions of radius $10 \mathrm{~mm}$. The size of this region was chosen because we used a spatial filter kernel of FWHM $=10 \mathrm{~mm}$, which roughly corresponds to the spatial scale of localization precision in group FMRI studies (Brett, Johnsrude, \& Owen, 2002; Petersson, Nichols, Poline, \& Holmes, 1999), including language (Hagoort, 2005; Petersson et al., 2004). In this analysis we used small volume correction based on the family-wise error (FWE) corrected for multiple non-independent comparisons based on smooth 3D random field theory (Adler \& Taylor, 2007).

\section{Results}

\subsection{Behavioral characterization}

Behavioral AGL results have previously been reported in Folia et al. (2008). Here we only give a brief summary of the most important results. Consistent with previous findings (Forkstam et al., 2006; Petersson et al., 2004) the overall correct classification performance was clearly above chance $(73 \pm 16 \%$ correct, $T(31)=7.7, P<.001)$. The analysis of hit rate showed that the subjects were sensitive to the grammaticality of the items $(F(2,62)=26, P<.001)$. Standard signal detection analysis showed a robust $d$-prime effect in discriminating between grammatical $(G)$ and non-grammatical (NG) sequences (mean $d$-prime: 1.53; $T(31)=7.63, P<.001)$. No significant response bias was found (mean beta-values: $1.17 ; P>.6$ ). Participants did not discriminate between high and low ACS sequences (mean $d$-prime: 0.21 ; $P>.66$ ), and no significant response bias was found (mean betavalues: $1.00 ; P>.8$ ). We then analyzed the performance data in terms of endorsement rate (i.e., item classified as grammatical, independent of their actual grammaticality status). In other words, if the subjects acquire significant aspects of the grammar, then they should endorse grammatical more often than non-grammatical items. Both grammaticality and local subsequence familiarity (ACS) influenced the endorsement rate. More specifically, the endorsement rate was significantly affected by grammaticality status $(F(1,31)=61.6, P<.001)$, and by $\operatorname{ACS}(F(1,31)=13.6, P<.001)$, while the interaction between grammaticality and ACS was nonsignificant $(F(1,31)=2.6, P=.11)$. In addition, we compared LG and HNG, because this comparison maximally contrasts structural vs. subsequence knowledge. If grammaticality status is used for classification, the acceptance of an LG item would crucially depend on the grammaticality of the item. If, in contrast, subsequence knowledge is used, the low ACS status would promote a rejection decision. On the other hand, if subsequence knowledge is used for classification, the acceptance of HNG items would depend on the high ACS status, while if grammatical status is used, the grammaticality status would indicate a rejection decision. We found a significant advantage for LG over HNG sequences in terms of endorsement rate (LG $>$ HNG; $T(31)=5.82, P<.001$ ). Taken together, these results show that, independent of local subsequence familiarity, grammaticality status is used for structural generalization in classifying novel sequences and thus provide support for the notion that grammatical structure instead of subsequence or fragment features determine classification.

\subsection{FMRI results}

Compared to the sensorimotor decision baseline, grammaticality classification significantly activated a set of regions $\left(P_{\mathrm{FWE}}<.001\right)$ similar to those found in previous studies (Forkstam et al., 2006; Petersson et al., 2004). These included the inferior and middle frontal regions bilaterally (cluster $P_{\mathrm{FWE}}<.001$ ), centered on BA 44/45 and extending into surrounding cortical regions (BA 6, 8, 9, 46, 47), including the frontal operculum and anterior insula (BA 13/15/47). Additional prefrontal activations included the anterior cingulate cortex (BA 32) bilaterally, extending laterally into anterior middle frontal regions (BA 6/8/9). Bilateral posterior activations (cluster $\left.P_{\mathrm{FWE}}<.001\right)$ included the inferior parietal cortex (BA 39/40), extending into the inferior parts of BA 7 and the posterior cingulate cortex (BA 24/31, cluster $P_{\mathrm{FWE}}<.001$ ) bilaterally. Bilateral occipital activations (cluster $P_{\mathrm{FWE}}<.001$ ) were centered on the middle and inferior occipital regions (BA 18/19) and extended into the fusiform (BA 19/37) and the posterior mid-inferior temporal (BA 20/21) regions, as well as the cerebellum. Significant activations were also observed in the basal ganglia bilaterally (cluster $P_{\mathrm{FWE}}<.001$ ), including the caudate nucleus, globus pallidus, putamen, and the medial aspects of the thalamus, extending into mesencephalic nuclei. Besides a typical default mode network, strong deactivations were found in the bilateral medial temporal lobe memory system, including the hippocampus proper (cluster $P_{\mathrm{FWE}}<.001$ ). The results were very similar when activity from the 
correctly classified HG- and LG sequences were compared separately with the sensorimotor decision baseline (Fig. 3).

As in previous studies (Forkstam et al., 2006; Petersson et al., 2004), artificial syntactic violations (main effect NG > G; Fig. 4) engaged a network of regions $\left(P_{\mathrm{FWE}}=.002\right)$, including the lateral prefrontal cortices bilaterally (left: cluster $P_{\mathrm{FWE}}<.001$; right: cluster $P_{\mathrm{FWE}}<.001$ ) centered on the inferior frontal region (BA 44/45), extending into BA 47 and the middle frontal cortex (BA 46), as well as frontal operculum/anterior insula (BA 13/15/47; cluster $\left.P_{\mathrm{FWE}}=.001\right)$. It further included regions in the anterior cingulate (BA 24/32; cluster $P_{\mathrm{FWE}}<.001$ ), the right inferior parietal (BA 39; cluster $P_{\mathrm{FWE}}<.001$ ), and the fusiform and inferior temporal (BA 19/20; cluster $\left.P_{\mathrm{FWE}}<.001\right)$ regions, bilaterally. As in previous studies (Folia et al., 2008; Forkstam, Elwér, Ingvar, \& Petersson, 2008; Forkstam et al., 2006), we examined the artificial syntactic violation effect $(\mathrm{NG}>G)$ by maximally contrasting structural knowledge vs. local subsequence familiarity (i.e., HNG vs. LG) in the lateral prefrontal cortex.

Consistent with previous findings, we observed significant effects in the left inferior frontal region (BA 44/45; cluster $\left.P_{\text {FWE }}<.001\right)$ while the right inferior frontal regions showed a trend $\left(P_{\mathrm{FWE}}=.063\right)$. In the reverse contrast $(G>N G)$, we observed a significant effect in the inferior medial frontal cortex (cluster $P_{\mathrm{FWE}}<0.001$ ), and we also replicated our previously reported caudate activation (Forkstam et al., 2006) with a small volume correction (centered at [3 $18-3$ ]; radius: $5 \mathrm{~mm}$; cluster $P_{\mathrm{FWE}}=.039$ ). There was no significant main effect of local subsequence familiarity (cluster $P_{\mathrm{FWE}}>.83$ ), neither were there any significant interactions (cluster $P_{\mathrm{FWE}}>.64$ ), consistent with our behavioral findings (Folia et al., 2008; Forkstam et al., 2008). We also tested LG > HG and HG > LG, but found no significant effect (cluster $P_{\mathrm{FWE}}>.95$ ).

Finally, we examined the common overlap between artificial and natural-syntax processing (Fig. 5) in the lateral prefrontal cortex by masking the NG vs. $G$ effect with the natural-syntax-related variability (i.e., the main effect of syntax in the $2 \times 2$ natural language experiment; cf., Table 1) observed in Folia et al. (2009, F-contrast thresholded at $P=.001$ ) in the same subjects. We examined these results in relation to recent claims of a syntactic specialization within Broca's region. We observed a common overlap in the left inferior frontal region (cluster $P_{\mathrm{FWE}}=.001$; including local maxima: [-50 2426$], \quad P_{\mathrm{FDR}}=.001 ; \quad\left[\begin{array}{lll}-48 & 8 & 34\end{array}\right], P_{\mathrm{FDR}}=.001 ; \quad[-462224]$, $P_{\mathrm{FDR}}=.001 ; \quad[-46828], \quad P_{\mathrm{FDR}}=.002 ; \quad[-42630], \quad P_{\mathrm{FDR}}=.002 ;$ [-50 16 30], $P_{\mathrm{FDR}}=.003$; cluster $P_{\mathrm{FWE}}=.003$; local maxima: [-30 24-2], $\left.P_{\mathrm{FDR}}=.001 ;[-4018-2], P_{\mathrm{FDR}}=.001\right)$ and right inferior frontal region (cluster $P_{\mathrm{FWE}}=.001$; local maxima: [36 24 -2],

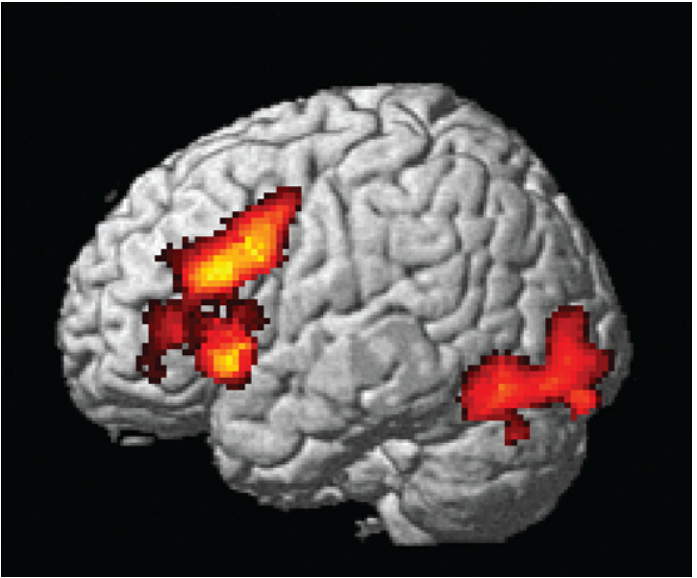

Fig. 4. Brain regions engaged during correctly classified artificial syntactic violations (i.e., main effect $N G>G$ ).

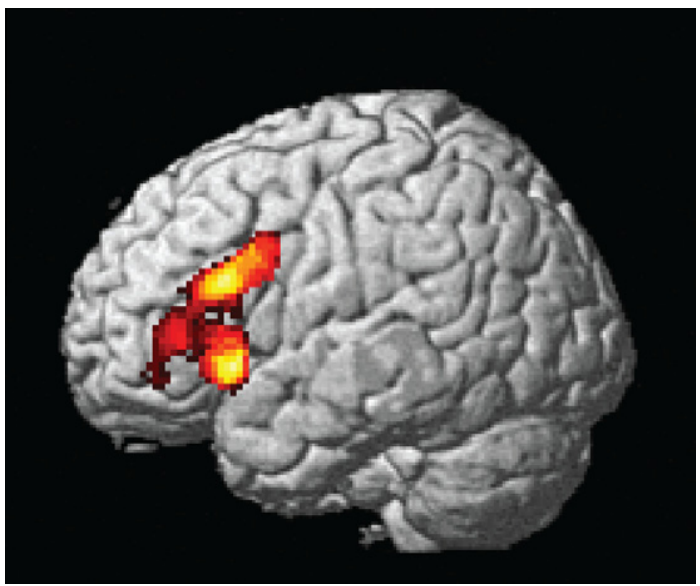

Fig. 5. Brain regions engaged during both artificial and natural-syntax processing The main effect NG > G masked with the syntax-related variability observed in Folia et al. (2009)

$\left.P_{\mathrm{FDR}}<.001 ;[32242], P_{\mathrm{FDR}}<.001\right)$, centered on BA 44 and 45 , as well as in the anterior cingulate (BA 32; cluster $P_{\mathrm{FWE}}<.001$ ). At the individual subject level, each subject showed overlap between artificial and natural-syntax processing in the left inferior frontal region, in or

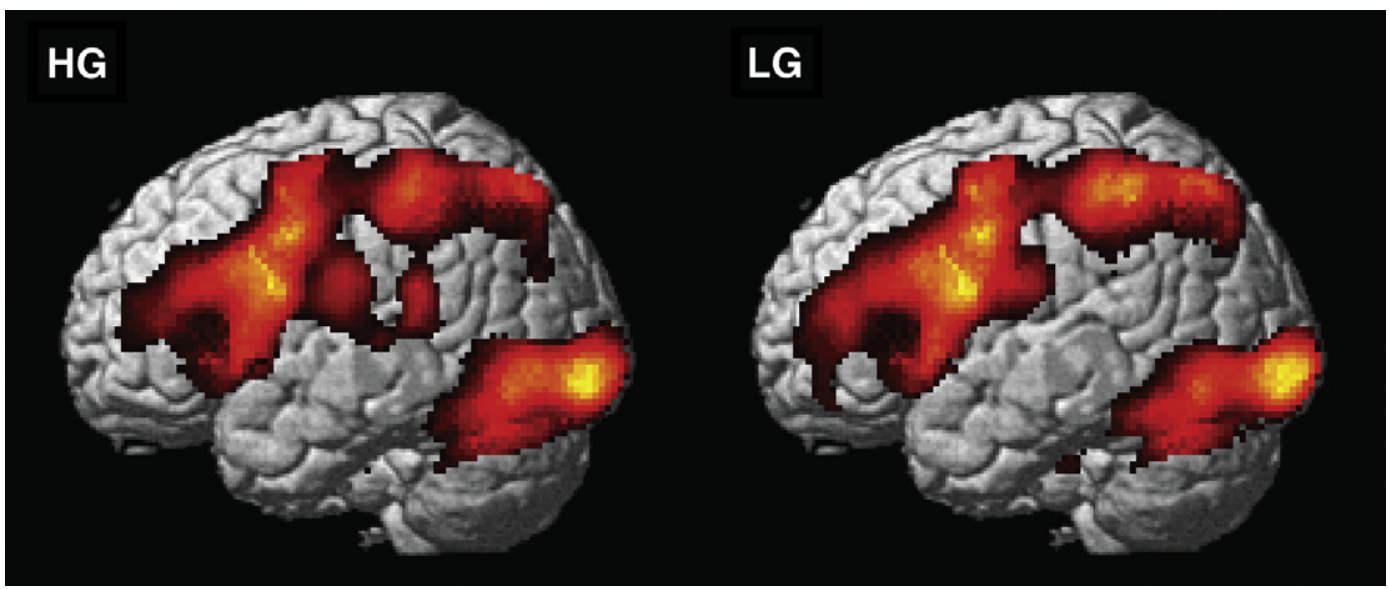

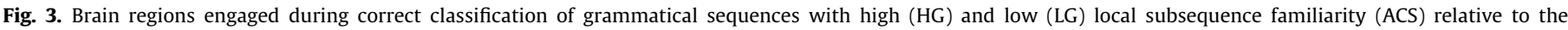
sensorimotor decision baseline. 
in the vicinity of BA 44/45. The localization of individual overlap varied from the posterior-superior parts to the anterior-inferior parts of the left inferior frontal gyrus (26 participants showed overlap in BA 44/45 and 5 in BA 47; $P<.001)$.

\section{Discussion}

In this study, the behavioral results convincingly show that the participants implicitly acquired significant knowledge about a simple unification grammar from grammatical examples alone and without performance feedback. The main FMRI results show that the left inferior frontal region, centered on BA 44 and 45 , is engaged during artificial syntax processing of well-formed (grammatical) sequences independent of local subsequence familiarity. This region is activated to a greater extent when the sequence contains an artificial syntactic violation, that is, when the unification of structural pieces becomes difficult or impossible. We note that the unification process is incremental and recursive even in its simplest instantiation (see Section 2/Section 2.3 for technical details). In addition, the effects related to artificial syntax processing in the left inferior frontal region (BA 44/45) were essentially identical when we masked these with activity related to natural-syntax processing in the same subjects. Our results are also highly consistent with functional localization of natural language syntax in the left inferior frontal gyrus (Bookheimer, 2002; Hagoort, 2005; Petersson et al., 2004). Similarly, Dominey and coworkers (Dominey \& Hoen, 2006; Hoen, Pachot-Clouard, Segebarth, \& Dominey, 2006), using a different approach based on construction grammars and explicit training of subjects on two constructions ("rules") for artificial and natural language materials, observed a common overlap between artificial and natural-language processing in several brain regions, including frontal regions. Finally, the medial temporal lobe was deactivated during artificial syntax processing, consistent with the view that implicit processing does not rely on declarative memory mechanisms that engage the medial temporal lobe memory system (Forkstam \& Petersson, 2005; Knowlton \& Squire, 1996; Seger, 1994).

How do these results square with some recently proposed functional roles of Broca's region or its subregions? For example, Grodzinsky and colleagues have claimed that Broca's region is specifically related to syntactic movement operations (Ben-Shachar, Hendler, Kahn, Ben-Bashat, \& Grodzinsky, 2003; Ben-Shachar, Palti, \& Grodzinsky, 2004; Santi \& Grodzinsky, 2007a, 2007b). Friederici and colleagues (2006) argue that their FMRI findings (p. 2458) "show that the processing of these two sequence types [generated by a "finite state" and a "phrase structure" grammar, respectively] is supported by different areas in the human brain" and that their results (p. 2460) "indicate a functional differentiation between two cytoarchitectonically and phylogenetically different brain areas in the left frontal cortex ["frontal operculum" and "BA 44"]". Similar reasoning is found in Friederici, Bahlmann et al. (2006), Friederici, Fiebach, Schlesewsky, Bornkessel, and von Cramon (2006), Bahlmann et al. (2008), and Makuuchi et al. (2009) ${ }^{1}$. In the context

\footnotetext{
${ }^{1}$ Bahlmann et al. (2008, p. 532): "This finding indicates that Broca's area is particularly engaged in processing hierarchical as compared to non-hierarchical grammars, a finding further supporting the idea formulated in a previous study (Friederici, Bahlmann et al. 2006", p. 533): "Rather, the notion put forward here is that Broca's area subserves the processing of hierarchical structures in the domain of grammar"; Makuuchi et al. (2009, p. 8362): "If the processing of PSG is fundamental to human language, the questions about how the brain implements this faculty arise. The left pars opercularis (LPO), a posterior part of Broca's area, was found as a neural correlate of the processing of $\mathrm{A}^{\mathrm{n}} \mathrm{B}^{\mathrm{n}}$ sequences in human studies by an artificial grammar learning paradigm comprised of visually presented syllables (Bahlmann et al., 2008; Friederici, Bahlmann et al. 2006", and p. 8367): these "2 studies therefore strongly suggest that LPO is a candidate brain area for the processor of PSG (i.e., hierarchical structures)".
}

of these FMRI findings and suggestions, we raise the question whether Broca's region (or subregions) is specifically related to syntactic movement operations or the processing of hierarchically nested non-adjacent dependencies? In Table 2 we specify the overlap between the clusters that we found activated in the left inferior frontal region in this study and the activated clusters reported in the above mentioned studies.

All regional effects of the studies reviewed here were localized within the left inferior frontal activation we observed in grammaticality classification as well as when only the correctly classified HG- and LG sequences were examined. This is clear from Fig. 3 showing the activity related to the correctly classified HG and LG sequences (left inferior frontal clusters centered on BA 44/45; cluster $\left.P_{\mathrm{FWE}}<.001\right)$. The only exception to this was [ -4265$]$ of Santi and Grodzinsky (2007a) where we find supra-threshold voxels localized $\sim 5 \mathrm{~mm}$ more laterally to that of Santi and Grodzinsky (2007a). This is because [-42 65] is localized to the superior part of mid-anterior insula, while our effect is localized to BA 44/45. Finally, the variability in spatial localization precision in the studies of Friederici et al. and Grodzinsky et al. were $\sim 13$ and $\sim 10 \mathrm{~mm}$, respectively. This is entirely consistent with what is known about localization precision in FMRI group studies (Brett et al., 2002; Petersson et al., 1999), including language (Bookheimer, 2002; Hagoort, 2005; Petersson et al., 2004), despite the fact that most of the reported coordinates were either chosen or observed in predetermined regions of interest. Our results show that the left inferior frontal region (BA 44/45) is significantly activated during artificial syntax processing, despite the fact that the simple rightlinear unification grammar we investigated does not involve syntactic movement or nested center-embeddings.

The main conclusion we draw from this comparison is that, in the context of language processing, the left inferior frontal region cannot be specific to the processing of syntactic movement or nested structures. Rather, our results, in conjunction with previous functional neuroimaging results, suggest that the left inferior frontal region is a generic on-line structured sequence processor that unifies information from various sources in an incremental and recursive manner (Hagoort, 2005, 2009), independent of whether there are any processing requirements related to syntactic movement or hierarchically nested structures. This is consistent with recent behavioral findings (Uddén et al., 2009, submitted for publication) that suggest that there is little qualitative differences between the implicit acquisition of non-adjacent and adjacent dependencies in humans. Taken together, we suggest that there is a quantitative difference (e.g., in terms of minimal memory requirements) in processing sequences with adjacent and nonadjacent dependencies, but that the nature of the underlying sequence processing is the same. This suggestion appears consistent with the position of Bahlmann et al. $(2008)^{2}$. Below, we review some of the literature supporting our position and then discuss the empirical findings in the light of some theoretical issues related to computability and the relevance of the Chomsky hierarchy for neurobiological mechanisms underlying syntax, since, in the final analysis, whatever operations linguists propose, these must ultimately be embedded in brain circuitry (Hornstein, 2009). However, first we make a few general remarks concerning the interpretation of functional neuroimaging results in the current context.

\footnotetext{
${ }^{2}$ Bahlmann et al. (2008) acknowledge (pp. 525-526) that the case of Friederici et al. (2006) might be overstated and that their own findings (p. 532) can be interpreted differently: "both rule types might be on the same level of the Chomsky hierarchy. Accordingly, the observed activation pattern in the contrast of hierarchical vs. adjacent dependency rule may not be taken to reflect grammatical complexity, but different dependency types involving different verbal working memory loads". To be fair, Bahlmann et al. (2008) never explicitly claim that Broca's region, including the subregion engaged by "hierarchical" sequences, is not involved in the processing of
} "non-hierarchical" sequences - the point we are substantiating in this paper. 
Table 2

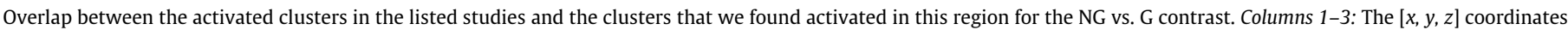

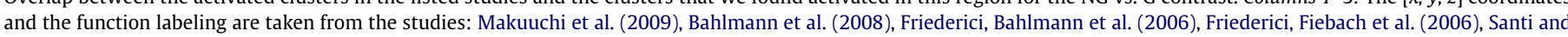

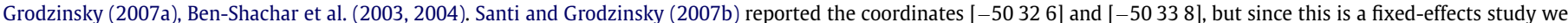

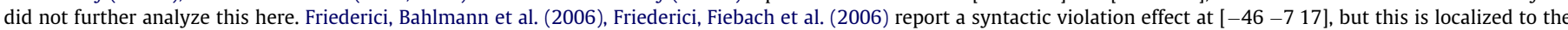

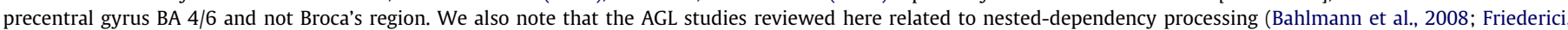

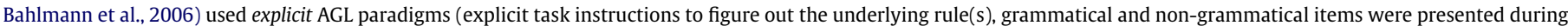

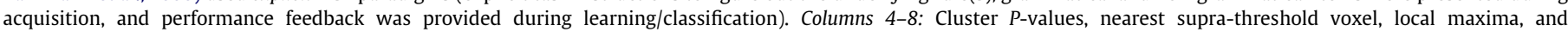
corresponding family-wise error rate corrected $P$-values $\left(P_{\text {FWE }}\right)$ are from the current study.

\begin{tabular}{|c|c|c|c|c|c|c|c|}
\hline Study & {$[x, y, z]$} & Function & Cluster & Nearest & $P_{\text {FWE }}$ & Local max & $P_{\text {FWE }}$ \\
\hline \multirow[t]{2}{*}{ Makuuchi et al. (2009) } & {$\left[\begin{array}{lll}-45 & 6 & 24\end{array}\right]$} & Nested & $P_{\mathrm{FWE}}=.001$ & {$\left[\begin{array}{lll}-46 & 6 & 26\end{array}\right]$} & .001 & {$\left[\begin{array}{lll}-4 & 8 & 32\end{array}\right]$} & .001 \\
\hline & & & & & & {$\left[\begin{array}{llll}-42 & 6 & 30\end{array}\right]$} & .002 \\
\hline \multirow[t]{2}{*}{ Bahlmann et al. (2008) } & {$\left[\begin{array}{llll}-4 & 5 & 16\end{array}\right]$} & Nested & $P_{\mathrm{FWE}}=.03$ & {$\left[\begin{array}{lll}-4 & 8 & 24\end{array}\right]$} & .001 & {$\left[\begin{array}{lll}-4 & 6 & 26\end{array}\right]$} & .02 \\
\hline & {$\left[\begin{array}{lll}-34 & 28 & 22\end{array}\right]$} & Nested & & {$\left[\begin{array}{lll}-42 & 26 & 20\end{array}\right]$} & .001 & {$\left[\begin{array}{lll}-42 & 22 & 22\end{array}\right]$} & .004 \\
\hline \multirow[t]{2}{*}{ Friederici Bahlmann et al. (2006) } & {$\left[\begin{array}{lll}-36 & 20 & -2\end{array}\right]$} & Nested & $P_{\mathrm{FWE}}<.001$ & {$\left[\begin{array}{lll}-36 & 20 & -2\end{array}\right]$} & .001 & {$\left[\begin{array}{lll}-30 & 24 & -2\end{array}\right]$} & .001 \\
\hline & {$\left[\begin{array}{lll}-46 & 16 & 8\end{array}\right]$} & Nested & & {$\left[\begin{array}{lll}-4 & 18 & 6\end{array}\right]$} & .001 & {$\left[\begin{array}{lll}-40 & 18 & 2\end{array}\right]$} & .001 \\
\hline Friederici Fiebach et al. (2006) & {$\left[\begin{array}{llll}-49 & 10 & 4\end{array}\right]$} & Complexity & $P_{\mathrm{FWE}}=.02$ & {$\left[\begin{array}{lll}-48 & 14 & 0\end{array}\right]$} & .001 & {$\left[\begin{array}{llll}-4 & 16 & 0\end{array}\right]$} & .001 \\
\hline Santi and Grodzinsky (2007a) & {$\left[\begin{array}{lll}-42 & 6 & 5\end{array}\right]$} & Movement & $P_{\mathrm{FWE}}<.05$ & {$\left[\begin{array}{lll}-42 & 16 & 6\end{array}\right]$} & .001 & {$\left[\begin{array}{lll}-42 & 16 & 6\end{array}\right]$} & .04 \\
\hline Ben-Shachar et al. (2003) & {$\left[\begin{array}{lll}-4 & 23 & 7\end{array}\right]$} & Movement & $P_{\mathrm{FWE}}=.005$ & {$\left[\begin{array}{lll}-46 & 24 & 6\end{array}\right]$} & .001 & {$\left[\begin{array}{lll}-42 & 20 & 0\end{array}\right]$} & .003 \\
\hline \multirow[t]{2}{*}{ Ben-Shachar et al. (2004) } & {$\left[\begin{array}{lll}-43 & 21 & 7\end{array}\right]$} & Movement & $P_{\mathrm{FWE}}=.004$ & {$\left[\begin{array}{lll}-44 & 22 & 6\end{array}\right]$} & .001 & {$\left[\begin{array}{lll}-40 & 20 & 0\end{array}\right]$} & .003 \\
\hline & {$\left[\begin{array}{llll}-4 & 21 & 8\end{array}\right]$} & Movement & & & & {$\left[\begin{array}{llll}-4 & 24 & 18\end{array}\right]$} & .05 \\
\hline
\end{tabular}

At a general level, unless one has an explicit neurobiological theory of for example the parsing process, it is seldom possible to determine with certainty which architectural component gives rise to a given observed effect. Experimental manipulations are typically described at Marr's level 1 or 2 (Marr, 1982), while the neurobiological observations are level 3 phenomena. Observed neurobiological effects, therefore, do not necessarily force a causal interpretation of results directly in terms of nominal experimental manipulations. More specifically, it would be surprising if very specific, and competence theory dependent linguistic phenomena, map onto brain regions or neocortical processes in a simple, specific, and transparent manner. For example, Hornstein (2009) argue that the view that language is a neurobiological system entails that the hypothesized linguistic constructs have to map onto neurophysiological processes, that is, recurrent spiking neural networks. It is known theoretically that (Church-Turing) computable processes can be embedded in the relevant type of dynamical systems instantiated by neural networks. However the relations between descriptive levels, implicit in this embedding, are not simple and transparent, but relies on intricate representational schemes (e.g., Buonomano \& Maass, 2009; Rabinovich, Varona, Selverston, \& Abarbanels, 2006; Siegelmann, 1999). However, for relatively transparent cases, see (e.g., Casey, 1996; Petersson, 2008; Rodriguez, 2001).

\subsection{Dynamic functional modularity and the role of the left inferior frontal region}

Claims that Broca's region, or more generally, that the left inferior frontal region, is specifically related to different aspects of language processing is not well supported by neuropsychological lesion studies nor by functional neuroimaging data. For example, Kaan and Swaab (2002) argue that lesion of Broca's region is neither necessary nor sufficient to induce syntactic deficits and suggest that Broca's aphasia can be interpreted as a processing deficit in contrast to a knowledge deficit. In other words, Broca's aphasia can be understood in terms of difficulties with certain aspects of temporal processing or on-line integration of information. Furthermore, several recent studies have shown that the left inferior frontal region has a broader role in cognition than just language (Hagoort, 2005; Marcus, Vouloumanos, \& Sag, 2003), including musical syntax (e.g., Koelsch et al., 2002; Maess, Koelsch, Gunter, \& Friederici, 2001) and visuo-spatial sequence processing (Bahlmann, Schubotz, Mueller, Koester, \& Friederici, in press). Thus, next to evidence from a study on aphasic patients (Patel,
Iversen, Wassenaar, \& Hagoort, 2008), a growing body of evidence from functional neuroimaging suggests an overlap in the processing of structural relations in language and music (for a review see Patel, 2003). It is also of interest to note that there seems to be a considerable overlap between regions implicated in the perception/production of music and the perception/production of abstract sequences, including the left inferior frontal region (Janata \& Grafton, 2003; Tillmann et al., 2006). Brain lesion data and results on specific language impairment (SLI) are consistent with these findings, suggesting that abnormal language processing are paralleled by impairment in structured sequence learning/processing (Christiansen, Kelly, Shillock, \& Greenfield, 2009; Evans, Saffran, \& Robe-Torres, 2009; Hoen et al., 2003; Hsu, Christiansen, Tomblin, Zhang, \& Gómez, 2006; Pothos \& Wood, 2009; Reali \& Christiansen, 2009; Richardson, Harris, Plante, \& Gerken, 2006; Uddén et al., 2008). However, there is a functional anterior-posterior gradient within the left inferior frontal region related to language (Bookheimer, 2002; Hagoort, 2005). The proposed gradient suggests that BA $45 / 47$ is related to unification of semantic structures (Folia et al., 2009; Hagoort, Hald, Bastiaansen, \& Petersson, 2004; Menenti, Petersson, Scheeringa, \& Hagoort, 2008; Tesink et al., 2008), BA $44 / 45$ to unification of syntactic structure (Folia et al., 2009; Snijders et al., 2009), and BA 6/44 to phonological unification (Bookheimer, 2002; see also Jackendoff, 2007).

There is also a growing body of evidence suggesting that regions other than Broca's region are related to the processing of syntactic information, such as the left superior anterior temporal lobe, the left middle and posterior parts of the superior and middle temporal gyri, as well as right-hemisphere regions (Snijders et al., 2009). Kaan and Swaab (2002) have argued that none of these regions are uniquely, or specifically, related to syntactic processing. It is thus not unreasonable to suggest that syntactic natural-language processing, or more generally the faculty of language, is in fact dependent on a functional network of interacting brain regions, none of which is uniquely and exclusively involved in syntactic processing (Hagoort, 2009).

One suggestion, therefore, is that particular brain regions are only computationally or processing specific in connection with other brain regions that provide the input from particular content domains. This notion gives rise to the concept of dynamic functional modularity, implying that the role of a given brain region at any given moment is determined in important respects by the neural processing context in which it participates - specifically, which other brain regions it currently interacts with. This notion is not new (Mesulam, 1990, 1998). In fact, dynamic functional 
modularity is the organizing principle for structured computing systems more generally, where processing and flow-control are implemented in the same hardware. This allows any given computing unit to take on various functional roles dynamically. For example, in the context of computers, the functional role of clusters of logical gates depends on the settings of control pins which in turn are recursively determined by earlier information processing (Savage, 1998; Tanenbaum, 1990). Therefore, we suggest, that dynamic functional modularity and neural processing context should be understood not only in terms of space (i.e., network interactions) but also in terms of time (i.e., processing history or memory). In other words, the function of a given brain region is to some extent dynamically determined by its full spatio-temporal processing context. As noted by Marcus et al. (2003), specific brain regions may genuinely participate in a range of tasks with specialized function emerging from unique network configurations of domain-general mechanisms. We conclude that the idea that Broca's region is, in any relevant sense, a syntax specific region is, overall, not well supported.

\subsection{Finiteness of neural systems}

Much of the discussion of the recursion-only hypothesis, concerning the narrow faculty of language (FLN, Hauser et al., 2002), has been phrased in terms of concepts derived from the Chomsky hierarchy; for example, non-regular context-free vs. right-linear regular grammars (e.g., de Vries, Monaghan, Knecht, \& Zwitserlood, 2008; Fitch \& Hauser, 2004; Gentner et al., 2006; Perruchet \& Rey, 2005; Uddén et al., 2009). Here, we will show why the Chomsky hierarchy is irrelevant in the context of finite processing systems. We take it as plausible that the brain is such a finite system. Beyond the finiteness of the processing infrastructure (e.g., finite number of logical gates, neurons, or finite machine tables), there are two concepts of finiteness that are relevant, depending on how one chooses to formalize computational models. The first, finiteness of memory, is relevant to classical cognitive models (i.e., Church-Turing computable), and the second, finiteness of processing precision, is relevant to non-classical computational models. We will discuss these matters mostly in terms of the machine formulation of the Chomsky hierarchy, instead of the equivalent grammar formulation (cf. e.g., Cutland, 1980; Davis et al., 1994; Hopcroft et al., 2000; Lewis \& Papadimitriou, 1981).

Cognitive neuroscience approaches the brain as a computational system - a system conceptualized in terms of information processing. This entails that a subclass of its physical states is viewed as representations and that transitions between states can be understood as a process implementing operations on the corresponding representational structures. It is uncontroversial that any physically realizable computational system is necessarily finite with respect to its memory organization and that it processes information with finite precision (e.g., presence of noise or architectural imprecision). We have previously indicated why this state of affairs implies the collapse of the Chomsky hierarchy for classical cognitive models, and why it makes the dynamical neurobiological analogue of the finite-state architecture relevant for non-classical computational models (Petersson, 2005b). Of course this does not imply that the Chomsky hierarchy is irrelevant for computational theory (Davis et al., 1994; Pullum \& Scholz, 2010), but as remarked in the introduction, the Chomsky hierarchy is in essence a memory hierarchy, specifying the necessary memory resources needed for a given level of expressivity. It is the infinite aspect inherent in the Chomsky hierarchy which makes it less relevant for neurobiological language research (Levelt, 1974; Petersson, 2005b; Pullum \& Scholz, 2010). However, bounded versions of the different memory architectures entailed by the hierarchy might be relevant (although we think these should not be taken too seriously). For example, one memory architecture in the hierarchy is the push-down stack, and it is conceivable that a bounded push-down stack is used in language processing, as suggested by for example Levelt (1974) as one possibility. We now turn to a brief discussion of both classical (Church-Turing) and non-classical computational architectures.

Turing (1936a, 1936b) explicitly models his machine on human calculation capacities in what must be some of the most important work in theoretical cognitive science (Wells, 2005). It clearly rivals the work of Chomsky $(1957,1965)$, who borrowed some of the crucial concepts and ideas from this branch of mathematics, including the work of Post (1944) and Kleene (1952). Turing explicitly designs the computational part of his machine as a finite-state control architecture for reasons of finite-precision computation (1936a; 1936b). What, then, is the crucial difference between the Turing machine architecture, which is at the top of the Chomsky hierarchy, and finite-state machines, which correspond to regular grammars? The answer is that, while finite-state machines have access to finite memory resources, Turing machines generally have infinite memory resources. Thus, Turing machines with finite memory resources are finite-state machines ${ }^{3}$ (see also e.g. ch. 1-4 in Savage, 1998; see also e.g. p.292 in Soare, 1996). It follows that any explicit classical cognitive model that takes into account the finite memory resources of the brain is necessarily a finite-state machine. In a fundamental sense, it is the characteristics of the memory organization that allow a computational architecture to re-use its processing capacities (i.e., computational mechanisms) recursively to generate its expressive range. If finite memory constraints are imposed, it follows that the computational mechanisms of universal architectures are no more powerful than that of the finite-state architecture. It is obvious that the finite-state architecture is the only computational architecture in the Chomsky hierarchy which is finite with respect to both its computational mechanism and its memory organization (Petersson, 2005b, 2008).

Given that the levels of the Chomsky hierarchy are strictly inclusive, it is commonly held that the finite-state architecture is too restrictive to capture all syntactic phenomena found in natural languages (Chomsky, 1956, 1957). However, as noted by for example Pullum and Scholz (2009), regular parsing is powerful. Pullum and Scholz (2009) argue that it would suffice for pretty much all the linguistic processing that humans ever do. Pullum and Scholz (2009) provide an interesting example of a regular language: $a\left(c^{*} d c^{*} d c^{*}\right)^{*} a+b\left(c^{*} d c^{*} d c^{*}\right)^{*} b$; in which all strings begin with either $a$ or $b$; the middle is an indefinitely long stretch of $c$ and $d$ in random order, but always containing an even number of d:s; and strings end with whatever they began with. Thus, in this regular language there are unbounded non-adjacent dependencies, and is parsed by a very simple finite-state machine. Pullum and Scholz (2009) argue that figuring out the underlying grammar from examples in this particular case would be way beyond the abilities of any mammal other than a skilled human puzzle-solver with pencil and paper.

Related to a different set of issues, Friederici (2004) claims that phrase-structure grammars are characterized by their admission of complex hierarchical structures and long-distance dependencies. In fact, András Kornai has provided one example of a context-sensitive grammar (there are infinitely many such generative grammars), with no useless rules - all rules participate

\footnotetext{
${ }^{3}$ It is trivial to see why this is the case. Consider a Turing machine with a finite memory tape. Include a given memory state $m$ (of which only a finite number exist by assumption) with the internal state $s$ of the finite-state control (of which only a finite number exist by definition) in an ordered pair $[s, m]$ (for all $s$ and $m$ ) and derive the corresponding machine table for the equivalent finite-state machine. Another simple example is a push-down machine with a stack memory of capacity $N<\infty$. An analogous argument applies in this case as well and yields the same conclusion.
} 
in all derivations that terminate - yet, only a finite string set is generated; and in the example of András Kornai, precisely one string is generated (cf., Pullum \& Scholz, 2010). In summary, regular grammars can represent non-adjacent dependencies, and conversely, there are non-trivial context-sensitive grammars that only generate a finite language. Therefore, the phenomenon of non-adjacent dependencies, a characteristic of natural languages, can not simply be reduced to a choice between regular or nonregular grammars.

From a neurobiological perspective, it is reasonable to assume that the brain only possesses finite memory resources (with respect to both short- and long-term memory). It is thus clear that seeing language as an internal system of the brain forces us to characterize language in terms of a computational system with finite memory resources. The argument here is simple: [neurobiological systems do not have greater capacity than any physically realizable information processor, i.e., finite-state capacity] \& [the language faculty is a neurobiological system] $\geq$ [the language faculty is finite-state system]. Moreover, finite-state and finite-precision computation devices, including real neural networks, are sufficient to handle finite recursion of general type, so there is no real problem here (Siegelmann, 1999; Siegelmann \& Fishman, 1998). It is also clear from, for example, Lasnik's Syntactic Structures Revisited (p. 4, 2000) that the assumption of infinite memory resources, $N=\infty$, is a simplifying assumption in theoretical linguistics (as in theoretical computer science), because the issue of memory limitations does not have to be dealt with explicitly although this is forced upon us when we deal with real computational mechanisms in brains (or real computers). It is also a common misconception that the finite-state architecture lacks memory, and that it is this limitation which prevents finite-state machines from successfully characterizing natural languages (e.g., Lasnik, 2000). On the contrary, the finite-state architecture supports a fixed finite memory resource (Minsky, 1967; Savage, 1998). We note that it is possible to implement any recursive type (general recursion) in a finite-state architecture as long as this is used with recursive depth $\leq N$, for some fixed $N<\infty$, or can be finitely expressed within this memory limitation during real-time operations. Thus, the finite-state architecture supports unlimited concatenation recursion and finite recursion of a general type and this is also the characteristic of human cognition and performance. In addition, these observations suggest an explanation why simple computational schemes are able to capture human performance qualitatively. For example, the discrete-time simple recurrent network (SRN) can be viewed as a simple network analogue of the finite-state architecture (Petersson, 2005b). Recent studies suggest that discrete-time SRNs, or similar network models, model different types of finite recursion with some success (Christiansen \& Chater, 1999; Elman, 1991; Misyak et al., 2009; Petersson, 2008; Petersson et al., 2005; Tong, Bickett, Christiansen, \& Cottrell, 2007).

The aforementioned arguments also imply that the fundamental difference between animal and human communication systems cannot be captured by direct reference to the Chomsky hierarchy, for example by referring to the distinction between the regular(e.g., right-linear or finite-state) and non-regular (e.g., context-free, context-sensitive) classes of grammars per se (e.g., Fitch \& Hauser, 2004; Gentner et al., 2006; Hauser et al., 2002). O'Donnell et al. (p. $287,2005)$, for example, notice that infinite ("unbounded") memory resources are necessary to process non-regular grammars. On the other hand, they state (p. 288, O'Donnell et al., 2005), "Fair tests of the generalization phase must of course, take into account factors such as known limitations on working memory in appropriate modalities". Taken together, this position does not seem coherent to us. We now turn to non-classical computational architectures.
Generally, analog dynamical systems provide a non-classical information processing alternative to classical computational architectures (Siegelmann \& Fishman, 1998). In particular, network approaches offer possibilities to model cognition within a nonclassical dynamical systems framework that is more natural from a brain perspective. As noted above, it is known theoretically that (Church-Turing) computable processes can be embedded in dynamical systems, instantiated by neural networks (e.g., Siegelmann, 1999). The recurrent neural network architecture can be viewed as a finite number of analog registers (e.g., the "membrane potential") that processes information interactively. Computations are determined by the network topology and by the transfer functions of the processing units, as well as the set of dynamical variables associated with the processing units. Several non-standard computational models have been outlined, including generalizations of the Church-Turing framework (for reviews see Buonomano \& Maass, 2009; Casey, 1996; Moore, 1990; Siegelmann, 1995, 1999; Siegelmann \& Fishman, 1998). However, their dependence on infinite precision processing implies that the computational capacities of these systems generally are sensitive to system noise. Theoretical results show that common types of noise put hard limits on the kinds of languages that analog neural networks can meaningfully process or recognize (Casey, 1996; Maass \& Orponen, 1997, 1998; Maass \& Sontag, 1999). We note that, in addition to external noise, there are several relevant brain-internal noise sources (e.g., Koch, 1999). Moreover, it seems clear that any reasonable analog model of a given brain system will have a statespace in the form of a finite dimensional compact manifold (i.e., closed and bounded). Here, compactness represents the natural generalization of finiteness in the classical framework. Qualitatively, it follows from the compactness property that finite processing precision or realistic noise levels have the effect of coarse graining the state-space - effectively "discretizing" this into a finite number of elements which become the relevant computational states. Thus, it seems that even if we model a given brain system as an analog dynamical system, this would approximately behave as, or could be well-approximated by, a finite-state analogue. Moreover, under the additional assumption that the available processing time is finite, the same conclusion follows for the continuous-time case, if finite temporal precision or temporal noise is assumed. This is essentially what the technical results of Maass and colleagues (Maass \& Orponen, 1997, 1998; Maass \& Sontag, 1999; Maass et al., 2007), as well as others (Casey, 1996; Siegelmann, 1999), entail.

The conclusions for non-classical computational frameworks are, therefore, essentially the same as for the classical framework. Thus, more than 70 years down the line, Turing's fundamental analysis of human cognition with explicit reference to finite-precision computation has proven to stand the tooth of time. Maass and colleagues (pp. 19-20, 2007) summarize these issues succinctly: "a generic neural circuit may become through feedback a universal computational device ... [and it is] ... known that the memory capacity of such a circuit is reduced to some finite number of bits if these feedback functions ... are not learnt perfectly [with infinite precision], or if there are other sources of noise in the system". They conclude that under noise or finite-precision computation, the maximal possible computational power that these systems achieve is that "they can simulate any given finite-state machine ... [and that] ... any Turing machine with tapes of finite length is a special case" (Maass et al., 2007).

What are the implications of this for theoretical models of language and grammar? If we view the faculty of language as a neurobiological system, given its finite storage capacity and finiteprecision computation, the Chomsky hierarchy is less relevant it does not make the relevant distinctions. The Chomsky hierarchy only has theoretical meaning in the context of infinite memory 
resources. Rather than giving "recursion" the centre stage, some of the real issues in the neurobiology of syntax, and language more generally, are related to the nature of the neural code (i.e., representation), the character of human on-line processing memory and neural finite-precision computation. Recurrent connectivity is a generic feature of brain network topology (Koch \& Laurent, 1999; Nieuwenhuys, Voogd, \& van Huijzen, 1988; Petersson, Ingvar, \& Reis, 2009). Thus, recursive processing is a latent capacity in almost any neurobiological system and it would be surprising, indeed, if this feature would be unique to the faculty of language. We noted that the relevant issue from the point of view of natural language is the human capacity to process patterns of non-adjacent dependencies - not arbitrarily "long" non-adjacent dependencies - there is a definite upper-bound set by the brain and its underlying neurophysiology. We can thus choose to work with any fruitful formal syntactic framework as long as this serves its purpose, for example, to capture the presence of relational patterns between lexical items in compositionally constructed sentences, or to elaborate a parameterized model of language acquisition. However, from a neurobiological perspective, it seems natural to try to understand language acquisition and language processing in terms of adaptive stochastic dynamical systems (Petersson, 2005a). Thus, the real challenge in the neurobiology of syntax is to understand syntax processing in terms of noisy spiking network processors. Similar, independent, accounts have been proposed by Culicover \& Nowak in their Dynamical Grammar (2003) as well as others (Christiansen \& Chater, 1999; Rodriguez, 2001; Rodriguez, Wiles, \& Elman, 1999).

\section{Conclusion}

We presented FMRI results on artificial syntax processing and showed that this engages the left inferior frontal region, centered on BA 44 and 45 , during the processing of well-formed sequences, independent of local subsequence familiarity. Crucially, the presented sequences lacked requirements for syntactic movement or nested embedding processing. We found that Broca's region is engaged to a greater extent when the system is attempting to process non-grammatical sequences, and thereby, the unification of structural pieces becomes difficult or impossible. These effects related to artificial syntactic processing were essentially identical when we masked these with activity related to natural-syntax processing in the same subjects. The behavioral performance of subjects paralleled the FMRI findings closely and were highly consistent with these.

In the context of recent FMRI findings related to Broca's region, we investigated whether this, or any of its subregions, can be considered specific to syntactic movement operations or the processing of hierarchically nested non-adjacent dependencies. We did so by comparing our results with findings interpreted in support for these functional roles of Broca's region. Essentially, we find that the same neocortical territory is engaged in structured sequence processing lacking such processing requirements. The main conclusion we draw from these findings is that the left inferior frontal region cannot be specific to the processing of syntactic movement or nested structures. Instead, our results, in conjunction with previous functional neuroimaging results, suggest that the left inferior frontal region is a generic on-line sequence processor that unifies information from various sources in an incremental and recursive manner, independent of whether there are any processing requirements related to syntactic movement or hierarchically nested structures. We suggest that there is a quantitative difference (e.g., in terms of minimal memory requirements) in processing sequences with adjacent and non-adjacent dependencies, but that the nature of the processing is the same. Finally, based on a theoretical review in combination with the notion of finiteness of neurobiological systems, we concluded that concepts derived from the Chomsky hierarchy are not directly relevant for neurobiological systems. This includes the theoretical distinction between regular and non-regular grammars. The relevant issue from the point of view of natural-language processing, is the human capacity to process bounded patterns of dependencies.

\section{Acknowledgement}

We are grateful to Morten Christiansen and Pienie Zwitserlood for their comments on a previous version of this paper. This work was supported by Max Planck Institute for Psycholinguistics, Donders Institute for Brain, Cognition and Behaviour, FCT grant IBB/ CBME, LA, FEDER/POCI 2010, Vetenskapsrådet (8276), Hedlunds Stiftelse and Stockholm County Council (ALF, FoUU). We thank Christian Forkstam for help with stimulus material and the experimental set-up.

\section{References}

Adler, R. J. (1981). The geometry of random fields. New York: Wiley and Sons. Adler, R. J., \& Taylor, J. E. (2007). Random fields and geometry. New York: Springer. Bahlmann, J., Schubotz, R. I., Mueller, J. L., Koester, D., \& Friederici, A. D. (in press). Neural circuits of hierarchical visuo-spatial sequence processing. Brain Research, doi:10.1016/j.brainres.2009.1008.1017.

Bahlmann, J., Schubotz, R. I., \& Friederici, A. D. (2008). Hierarchical artificial grammar processing engages Broca's area. Neurolmage, 42, 525-534.

Ben-Shachar, M., Hendler, T., Kahn, I., Ben-Bashat, D., \& Grodzinsky, Y. (2003). The neural reality of syntactic transformations: Evidence from functional magnetic resonance imaging. Psychological Science, 14, 433-440.

Ben-Shachar, M., Palti, D., \& Grodzinsky, Y. (2004). Neural correlates of syntactic movement: Converging evidence from two fMRI experiments. NeuroImage, 21, 1320-1336.

Bookheimer, S. (2002). Functional MRI of language: New approaches to understanding the cortical organization of semantic processing. Annu. Rev. Neurosci., 25, 151-188.

Brett, M., Johnsrude, I. S., \& Owen, A. M. (2002). The problem of functional localization in the human brain. Nat. Rev. Neurosci., 3, 243-249.

Buonomano, D. V., \& Maass, W. (2009). State-dependent computations: Spatiotemporal processing in cortical networks. Nature Reviews Neuroscience, $10,113-125$.

Casey, M. (1996). The dynamics of discrete-time computation with application to recurrent neural networks and finite state machine extraction. Neural Computation, 8, 1135-1178.

Chomsky, N. (1956). Three models for the description of language. IEEE Transactions on Information Theory, 2, 113-124.

Chomsky, N. (1957). Syntactic Structures. Den Haag: Mouton.

Chomsky, N. (1965). Aspects of the theory of syntax. Cambridge, MA: MIT Press.

Chomsky, N., Fitch, W. T., \& Hauser, M. D. (2005). The evolution of the language faculty: Clarifications and implications. Cognition, 97, 179-210.

Christiansen, M. H., \& Chater, N. (1999). Toward a connectionist model of recursion in human linguistic performance. Cognitive Science, 23, 157-205.

Christiansen, M. H., Kelly, L., Shillock, R., \& Greenfield, K. (2009). Impaired artificial grammar learning in agrammatism. Unpublished manuscript.

Christiansen, M. H., \& MacDonald, M. C. (2009). A usage-based approach to recursion in sentence processing. Language Learning, 59(Suppl. 1), 126-161.

Culicover, P. W., \& Nowak, A. (2003). Dynamical Grammar: Volume two of foundations of syntax. Oxford, UK: Oxford University Press.

Cutland, N. J. (1980). Computability: An introduction to recursive function theory. Cambridge, UK: Cambridge University Press.

Davis, M., Sigal, R., \& Weyuker, E. J. (1994). Computability, complexity, and languages: Fundamentals of Theoretical Computer Science. San Diego CA: Academic Press.

de Vries, M. H., Monaghan, P., Knecht, S., \& Zwitserlood, P. (2008). Syntactic structure and artificial grammar learning: The learnability of embedded hierarchical structures. Cognition, 107, 763-774.

Dominey, P. F., \& Hoen, M. (2006). Structure mapping and semantic integration in a construction-based neurolinguistic model of sentence processing. Cortex, 42 , 476-479.

Elman, J. (1991). Distributed representations, simple recurrent networks, and grammatical structure. Machine Learning, 7, 195-224.

Evans, J. L., Saffran, J. R., \& Robe-Torres, K. (2009). Statistical learning in children with specific language impairment. J. Speech. Lang. Hear. Res., 52, 321-335.

Fitch, W. T., \& Hauser, M. D. (2004). Computational constraints on syntactic processing in a nonhuman primate. Science, 303, 377-380.

Folia, V., Forkstam, C., Hagoort, P., \& Petersson, K. M. (2009). Language comprehension: The interplay between form and content. Proceedings of the Cognitive Science Society, 2009, 1686-1691. 
Folia, V., Uddén, J., Forkstam, C., Ingvar, M., Hagoort, P., \& Petersson, K. M. (2008). Implicit learning and dyslexia. Annals of the New York Academy of Sciences, 1145, 132-150.

Forkstam, C., Elwér, Å., Ingvar, M., \& Petersson, K. M. (2008). Instruction effects in implicit artificial grammar learning: A preference for grammaticality. Brain Research, 1221, 80-92.

Forkstam, C., Hagoort, P., Fernandez, G., Ingvar, M., \& Petersson, K. M. (2006). Neural correlates of artificial syntactic structure classification. Neurolmage, 32, 956-967.

Forkstam, C., \& Petersson, K. M. (2005). Towards an explicit account of implicit learning. Current Opinion in Neurology, 18, 435-441.

Friederici, A. D. (2004). Processing local transitions vs. long-distance syntactic hierarchies. Trends in Cognitive Sciences, 8, 245-247.

Friederici, A. D., Bahlmann, J., Heim, S., Schubotz, R. I., \& Anwander, A. (2006). The brain differentiates human and non-human grammars: Functional localization and structural connectivity. Proceedings of the National Acadamic Science USA, 103, 2458-2463.

Friederici, A. D., Fiebach, C. J., Schlesewsky, M., Bornkessel, I. D., \& von Cramon, D. Y. (2006). Processing linguistic complexity and grammaticality in the left frontal cortex. Cerebral Cortex, 16, 1709-1717.

Friston, K. J. (1997). Testing for anatomically specified regional effects. Human Brain Mapping, 5, 133-136.

Friston, K. J., Ashburner, J. T., Kiebel, S. J., Nichols, T. E., \& Penny, W. D. (Eds.). (2007). Statistical parametric mapping: The analysis of functional brain images. San Diego, CA: Academic Press.

Genovese, C. L., Lazar, N. A., \& Nichols, T. (2002). Thresholding of statistical maps in functional neuroimaging using the false discovery rate. Neurolmage, 15, 870-878.

Gentner, T. Q., Fenn, K. M., Margoliash, D., \& Nusbaum, H. C. (2006). Recursive syntactic pattern learning by songbirds. Nature, 440, 1204-1207.

Gómez, R. L., \& Gerken, L. (2000). Infant artificial language learning and language acquisition. Trends in Cognitive Sciences, 4, 178-186.

Hagoort, P. (2003). Interplay between syntax and semantics during sentence comprehension: ERP effects of combining syntactic and semantic violations. Journal of Cognitive Neuroscience, 15, 883-899.

Hagoort, P. (2005). On Broca, brain, and binding: A new framework. Trends in Cognitive Sciences, 9, 416-423.

Hagoort, P. (2009). Reflections on the neurobiology of syntax. In XXX (Ed.), Biological foundations and origin of syntax (pp. 1-27). Cambridge, MA: MIT Press.

Hagoort, P., Hald, L., Bastiaansen, M., \& Petersson, K. M. (2004). Integration of word meaning and world knowledge in language comprehension. Science, 304, 438-441.

Hauser, M. D., Chomsky, N., \& Fitch, W. T. (2002). The faculty of language: What is it, who has it, and how did it evolve? Science, 298, 1569-1579.

Hoen, M., Golembiowski, M., Guyot, E., Deprez, V., Caplan, D., \& Dominey, P. F. (2003). Training with cognitive sequences improves syntactic comprehension in agrammatic aphasics. NeuroReport, 14, 495-499.

Hoen, M., Pachot-Clouard, M., Segebarth, C., \& Dominey, P. F. (2006). When Broca experiences the Janus syndrome: An ER-fMRI study comparing sentence comprehension and cognitive sequence processing. Cortex, 42, 605-623.

Hopcroft, J. E., Motwani, R., \& Ullman, J. D. (2000). Introduction to automata theory languages, and computation. Reading. MA: Addison-Wesley.

Hornstein, N. (2009). A theory of syntax: Minimal operations and universal grammar. Cambridge, UK: Cambridge University Press.

Hsu, H. -J., Christiansen, M. H., Tomblin, J. B., Zhang, X., \& Gómez, R. L. (2006) Statistical learning of nonadjacent dependencies in adolescents with and without language impairment. In Symposium on research in child language disorders. Madison, WI.

Indefrey, P., \& Levelt, W. J. M. (2004). The spatial and temporal signatures of word production components. Cognition, 92, 101-144.

Jackendoff, R. (2007). A parallel architecture perspective on language processing. Brain Research, 1146, 2-22.

Jackendoff, R., \& Pinker, S. (2005). The nature of the language faculty and its implications for evolution of language. Cognition, 97, 211-225.

Janata, P., \& Grafton, S. T. (2003). Swinging in the brain: Shared neural substrates for behaviors related to sequencing and music. Nature Neuroscience, 6, 682-687.

Joshi, A. K., \& Schabes, Y. (1997). Tree-adjoining grammars. In A. Salomaa (Ed.). Handbook of formal languages (Vol. 3: Beyond words, pp. erlag). Berlin: Springer.

Kaan, E., \& Swaab, T. Y. (2002). The brain circuitry of syntactic comprehension. Trends in Cognitive Sciences, 6, 350-356.

Kleene, S. (1952). Introduction to metamathematics. NewYork: Van Nostrand.

Knowlton, B. J., \& Squire, L. R. (1996). Artificial grammar learning depends on implicit acquisition of both abstract and exemplar-specific information. Journal of Experimental Psychology Learning Memory Cognition, 22, 169-181.

Koch, C. (1999). Biophysics of computation: Information processing in single neurons. New York: Oxford University Press.

Koch, C., \& Laurent, G. (1999). Complexity and the nervous system. Science, 284, 96-98.

Koelsch, S., Gunter, T. C., von Cramon, D. Y., Zysset, S., Lohmann, G., \& Friederici, A. D. (2002). Bach speaks: A cortical "language-network" serves the processing of music. Neurolmage, 17, 956-966.

Lasnik, H. (2000). Syntactic structures revisited: Contemporary lectures on classic transformational theory. Cambridge, MA: MIT Press.

Levelt, W. J. M. (1974). Formal grammars in linguistics and psycholinguistics (Vol. III). The Hague: Mouton.
Lewis, H., \& Papadimitriou, C. H. (1981). Elements of the theory of computation. Upper Saddle River, NJ: Prentice Hall.

Maass, W., Joshi, P., \& Sontag, E. (2007). Computational aspects of feedback in neural circuits. PLoS Computational Biology, 3(1), e165. doi:110.1371/ journal.pcbi.0020165.

Maass, W., \& Orponen, P. (1997). On the effect of analog noise on discrete-time analog computations. Advances in Neural Information Processing Systems, 9 218-224.

Maass, W., \& Orponen, P. (1998). On the effect of analog noise on discrete-time analog computations. Neural Computation, 10, 1071-1095.

Maass, W., \& Sontag, E. (1999). Analog neural nets with Gaussian or other common noise distribution cannot recognize arbitrary regular languages. Neural Computation, 11, 771-782.

Maess, B., Koelsch, S., Gunter, T. C., \& Friederici, A. D. (2001). Musical syntax is processed in Broca's area: A MEG study. Nature Neuroscience, 4, 540-545.

Makuuchi, M., Bahlmann, J., Anwander, A., \& Friederici, A. D. (2009). Segregating the core computational faculty of human language from working memory. Proceedings of the National Acadamic Science USA, 106, 8362-8367.

Marcus, G. F., Vouloumanos, A., \& Sag, I. A. (2003). Does Broca's play by the rules? Nature Neuroscience, 6, 651-652.

Marr, D. (1982). Vision: A computational investigation into the human representation and processing of visual information. New York: W. H. Freeman and Company.

Menenti, L., Petersson, K. M., Scheeringa, R., \& Hagoort, P. (2008). When elephants fly: Differential sensitivity of right and left inferior frontal gyri to discourse and world knowledge. Journal of Cognitive Neuroscience.. doi: 10.1162 jocn.2008.21163.

Mesulam, M.-M. (1990). Large-scale neurocognitive networks and distributed processing for attention, language, and memory. Annals of Neurology, 28 597-613.

Mesulam, M.-M. (1998). Form sensation to cognition. Brain, 121, 1013-1052.

Meulemans, T., \& Van der Linden, M. (1997). Associative chunk strength in artificial grammar learning. Journal of Experimental Psychology Learning Memory Cognition, 23, 1007-1028.

Minsky, M. L. (1967). Computation: Finite and infinite machines. Englewood Cliffs, NJ: Prentice-Hall.

Misyak, J. B., Christiansen, M. H., \& Tomblin, J. B. (2009). Statistical learning of nonadjacencies predicts on-line processing of long-distance dependencies in natural language. Proceedings of the Cognitive Science Society, 2009, 177-182.

Misyak, J. B., Christiansen, M. H., \& Tomblin, J. B. (2010). Sequential expectations: The role of prediction-based learning in language. Topics in Cognitive Science, 2, 138-153.

Moore, C. (1990). Unpredictability and undecidability in dynamical systems Physical Review Letters, 64, 2354-2357.

Nieuwenhuys, R., Voogd, J., \& van Huijzen, C. (1988). The human central nervous system: A synopsis and atlas (3rd revised ed.). Berlin: Springer-Verlag.

O'Donnell, T. J., Hauser, M. D., \& Fitch, W. T. (2005). Using mathematical models of language experimentally. Trends in Cognitive Sciences, 9, 284-289.

Patel, A. D. (2003). Language, music, syntax and the brain. Nature Neuroscience, 6 674-681.

Patel, A. D., Iversen, J., Wassenaar, M., \& Hagoort, P. (2008). Musical syntactic processing in agrammatic Broca's aphasia. Aphasiology, 22, 776-789.

Perruchet, P., \& Rey, A. (2005). Does the mastery of center-embedded linguistic structures distinguish humans from nonhuman primates? Psychonomic Bulletin E Review, 12, 307-313.

Petersson, K. M. (2005a). Learning and memory in the human brain. Stockholm, Sweden: Karolinska University Press.

Petersson, K. M. (2005b). On the relevance of the neurobiological analogue of the finite-state architecture. Neurocomputing, 65-66, 825-832.

Petersson, K. M. (2008). On cognition, structured sequence processing and adaptive dynamical systems. Proceedings of the American Institute of Physics: Mathematical and Statistical Physics Subseries, 1060, 195-200.

Petersson, K. M., Forkstam, C., \& Ingvar, M. (2004). Artificial syntactic violations activate Broca's region. Cognitive Science, 28, 383-407.

Petersson, K. M., Grenholm, P., \& Forkstam, C. (2005). Artificial grammar learning and neural networks. Proceeding of the Cognitive Science Society, 2005, 1726-1731.

Petersson, K. M., Ingvar, M., \& Reis, A. (2009). Language and literacy from a cognitive neuroscience perspective, Cambridge Handbook of Literacy. Cambridge, UK: Cambridge University Press (pp. 152-182).

Petersson, K. M., Nichols, T. E., Poline, J.-B., \& Holmes, A. P. (1999). Statistica limitations in functional neuroimaging II: Signal detection and statistica inference. Philosophical Transactions of the Royal Society B, 354, 1261-1282.

Pinker, S., \& Jackendoff, R. (2005). The faculty of language: what's special about it? Cognition, 95, 201-236.

Poletiek, F. H. (2002). Implicit learning of a recursive rule in an artificial grammar. Acta Psychologica, 111, 323-335.

Post, E. (1944). Recursively enumerable sets of positive integers and their decision problems. Bulletin of the American Mathematical Society, 50, 284-316.

Pothos, E. M., \& Wood, R. L. (2009). Separate influences in learning: Evidence from artificial grammar learning with traumatic brain injury patients. Brain Research $1275,67-72$.

Pullum, G. K., \& Scholz, B. C. (2009). For universals (but not finite-state learning) visit the zoo. Behavioral and Brain Sciences, 32, 466-467.

Pullum, G. K., \& Scholz, B. C. (2010). Recursion and the infinitude claim. In H. van der Hulst (Ed.), Recursion in human language. Berlin: Mouton de Gruyter. pp. 1-9. 
Rabinovich, M. I., Varona, P., Selverston, A. I., \& Abarbanels, H. D. I. (2006) Dynamical principles in neuroscience. Reviews of Modern Physics, 78, 1214-1265.

Reali, F., \& Christiansen, M. H. (2009). Sequential learning and the interaction between biological and linguistic adaptation in language evolution. Interaction Studies, 10, 5-30.

Reber, A. S. (1967). Implicit learning of artificial grammars. Journal of Verbal Learning and Verbal Behavior, 5, 855-863.

Richardson, J., Harris, L., Plante, E., \& Gerken, L. (2006). Subcategory learning in normal and language learning-disabled adults: How much information do they need? Journal of Speech Language and Hearing Research, 49, 1257-1266.

Rodriguez, P. (2001). Simple recurrent networks learn context-free and contextsensitive languages by counting. Neural Computation, 13, 2093-2118.

Rodriguez, P., Wiles, J., \& Elman, J. L. (1999). A recurrent neural network that learns to count. Connection Science, 11, 5-40.

Saffran, J. R., Hauser, M., Seibel, R., Kapfhamer, J., Tsao, F., \& Cushman, F. (2008) Grammatical pattern learning by human infants and cotton-top tamarin monkeys. Cognition, 107, 479-500.

Santi, A., \& Grodzinsky, Y. (2007a). Taxing working memory with syntax: Bihemispheric modulation. Human Brain Mapping, 28, 1089-1097.

Santi, A., \& Grodzinsky, Y. (2007b). Working memory and syntax interact in Broca's area. Neurolmage, 37, 8-17.

Savage, J. E. (1998). Models of Computation. Reading, MA: Addison-Wesley.

Seger, C. A. (1994). Implicit learning. Psychonomic Bulletin, 115, 163-196.

Siegelmann, H. T. (1995). Computation beyond the turing limit. Science, 268, 545-548.

Siegelmann, H. T. (1999). Neural networks and analog computation: Beyond the turing limit. Basel: Birkhäuser.

Siegelmann, H. T., \& Fishman, S. (1998). Analog computation with dynamical systems. Physica D, 120, 214-235.

Snijders, T. M., Vosse, T., Kempen, G., Van Berkum, J. A., Petersson, K. M., \& Hagoort P. (2009). Retrieval and unification in sentence comprehension: An fMRI study using word-category ambiguity. Cerebral Cortex, 19, 1493-1503.

Soare, R. I. (1996). Computability and recursion. Bulletin of Symbolic Logic, 2, 284-321.

Stadler, M. A., \& Frensch, P. A. (Eds.). (1998). Handbook of implicit learning. London: SAGE.

Talairach, J., \& Tournoux, P. (1988). Co-planar stereotaxic atlas of the human brain. New York: Thieme Verlag.
Tanenbaum, A. S. (1990). Structured computer organization. Englewood Cliffs, NJ: Prentice-Hall.

Tesink, C. M. J. Y., Petersson, K. M., Van Berkum, J. A., van den Brink, D., Buitelaar, J. K., \& Hagoort, P. (2008). Unification of speaker and meaning in language comprehension: An fMRI study. Journal of Cognitive Neuroscience. doi: 10.1162/ jocn.2008.21161.

Tillmann, B., Koelsch, S., Escoffier, N., Bigand, E., Lalitte, P., Friederici, A. D., et al. (2006). Cognitive priming in sung and instrumental music: Activation of inferior frontal cortex. Neurolmage, 31, 1771-1782.

Tong, M. H., Bickett, A. D., Christiansen, E. M., \& Cottrell, G. W. (2007). Learning grammatical structure with Echo State Networks. Neural Networks, 20, 424-432.

Turing, A. (1936a). On computable numbers with an application to the Entscheidungs problem (part 1). Proceedings of the London Mathematical Society, 42(3), 230-240.

Turing, A. (1936b). On computable numbers with an application to the Entscheidungs problem (part 2). Proceedings of the London Mathematical Society, 42(4), 241-265.

Uddén, J., Araujo, S., Forkstam, C., Ingvar, M., Hagoort, P., \& Petersson, K. M. (2009). A matter of time: Implicit acquisition of recursive sequence structures. Proceedings of the Cognitive Science Society, 2009, 2444-2449.

Uddén, J., Folia, V., Forkstam, C., Ingvar, M., Fernandez, G., Overeem, S., et al. (2008) The inferior frontal cortex in artificial syntax processing: An rTMS study. Brain Research, 1224, 69-78.

Uddén, J., Ingvar, M., Hagoort, P., \& Petersson, K. M. (submitted for publication). Implicit acquisition of grammars with crossed and nested non-adjacent dependencies: Investigating the push-down stack model.

Vosse, T., \& Kempen, G. (2000). Syntactic structure assembly in human parsing: A computational model based on competitive inhibition and a lexicalist grammar. Cognition, 75, 105-143.

Wells, A. (2005). Rethinking cognitive computation: Turing and the science of the mind. Hampshire, UK: Palgrave Macmillan.

Worsley, K. J. (2003). Developments in random field theory. In R. S. J. Frackowiak, K J. Friston, C. Frith, R. Dolan, C. Price, S. Zeki, J. Ashburner, \& W. D. Penny (Eds.), Human Brain Function (pp. 881-886). San Diego, CA: Academic Press.

Worsley, K. J., Marrett, S., Neelin, P., Vandal, A. C., Friston, K. J., \& Evans, A. C. (1996). A unified statistical approach for determining significant signals in images of cerebral activation. Human Brain Mapping, 4, 58-73. 